\title{
Vibrations des directrices des turbomachines à l'emballement
}

\author{
Vibrations of guide vanes of turbomachinery \\ in runaway operation
}

\author{
P. Boussuges, $\mathbb{H}$. Amblard et $\mathbb{C}$. Lathuile \\ Neyrpic Grenoble
}

Les sollicitations que subissent les directrices mobiles des turbomachines (turbines-pompes et turbines) au cours des divers régimes de fonctionnement, ont déjà fait l'objet de plusieurs communications lors de précédentes sessions.

A partir d'essais sur modèle et sur machine industrielle, l'étude actuelle se limite au cas de l'emballement turbine, au cours duquel les directrices sont le plus fortement sollicitées.

Ce régime est souvent atteint au cours de l'exploitation, soit d'une manière transitoire lors des déclenchements, soit d'une façon permanente pendant les marches à vide.

Après avoir analysé les pulsations de pression, dans l'entrefer roue-distributeur, induites par la roue pour des machines de différentes vitesses spécifiques, nous examinerons les fluctuations de couple sur les directrices.

Nous commençons tout d'abord par présenter une schématisation de l'écoulement dans la roue à l'emballement.

Nous avons porté sur la figure 1 , les courbes de répartition des pressions sur une aube à ce régime. Remarquons qu'il s'agit de l'image d'un écoulement permanent en fluide parfait. Ces courbes ont été obtenues de la façon suivante en utilisant un programme d'écoulement pseudotridimensionnel.

- l'ouverture du distributeur étant fixée, la circulation le long de l'aube est calculée pour deux débit $Q_{1}$ et $Q_{2}$ au voisinage du point nominal de fonctionnement. La droite de hauteur interne en fonction du débit $H_{i}(Q)$ s'en déduit. L'abscisse à l'origine $\left(H_{i}=0\right)$ détermine le débit théorique à l'emballement ;

- ce modèle mathématique permet d'obtenir la distribution des vitesses autour du profil pour un débit quelconque $Q$, au moyen d'une combinaison linéaire des vitesses calculées pour les deux débits $Q_{1}$ et $Q_{2}$ à la même ouverture du distributeur.
La répartition des vitesses calculées, pour le débit théorique d'emballement, permet de déduire la répartition des pressions.

Il est ensuite possible de vérifier, en faisant l'intégration des pressions, que le couple sur l'arbre est bien nul au régime considéré.

La figure 1 fait apparaître un ralentissement considérable de la vitesse sur la face $(a)$ du profil. C'est ce ralentissement qui provoque des décollements importants dans cette zone et donne naissance à d'importantes fluctuations de pression dans l'entrefer roue-distributeur.

\section{Fluctuations de pression dans l'entrefer}

Examinons le rapport de l'amplitude des fluctuations de couple et des fluctuations de pression dans l'entrefer. En exprimant, suivant les fréquences, l'évolution respective des modules de la fonction de transfert du rapport précédent et de la fonction de cohérence, nous obtenons les courbes figures 2 et 3 qui correspondent aux essais des machines de Ste Croix, de Revin et du Tage.

L'examen de ces deux courbes montre une cohérence maximum (voisine de 1) pour la fréquence de défilement des aubes de la roue et de son premier harmonique.

Le fait que la cohérence soit moins élevée en dehors de ces zones s'explique probablement par la différence de nature des deux paramètres considérés. L'un, le capteur de pression, donne une mesure locale ; l'autre, le couple, représente une intégration de pression le long du profil. De toute manière, le résultat de cette analyse montre une dépendance effective "couple-pression" pour les régimes considérés. C'est cette dépendance "couple-pression" qui nous a conduit à analyser dans un premier temps les fluctuations de pression. 


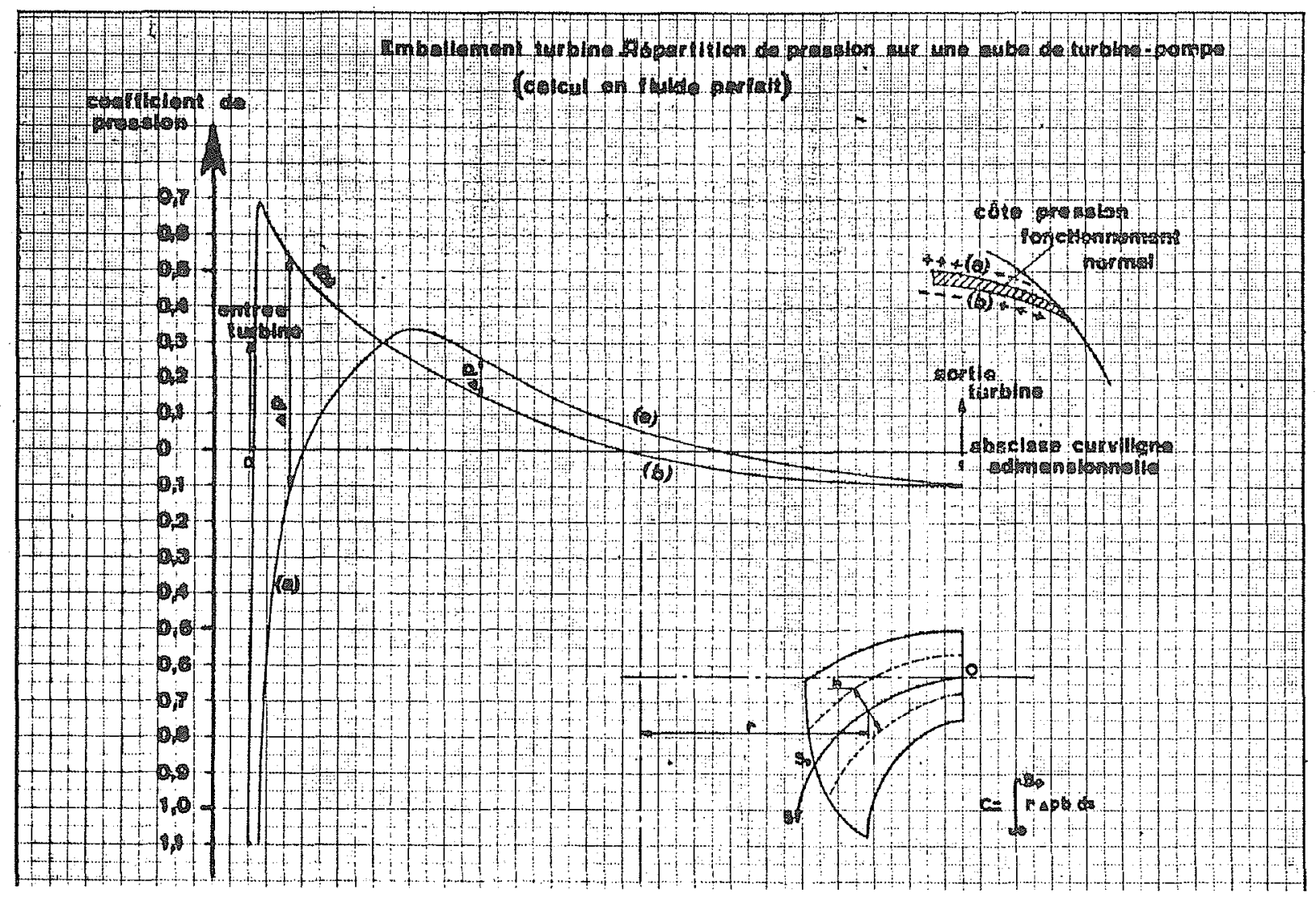

Figure 1 - Emballement turbine. Répartition de pression sur une aube de turbine-pompe (calcul en fluide parfait).
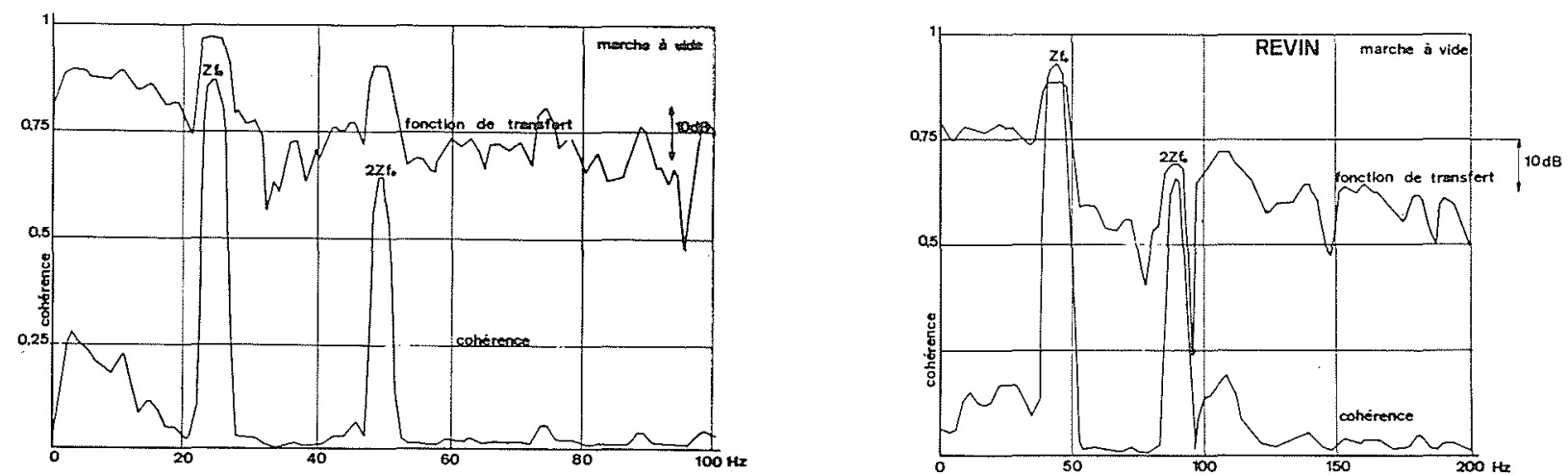

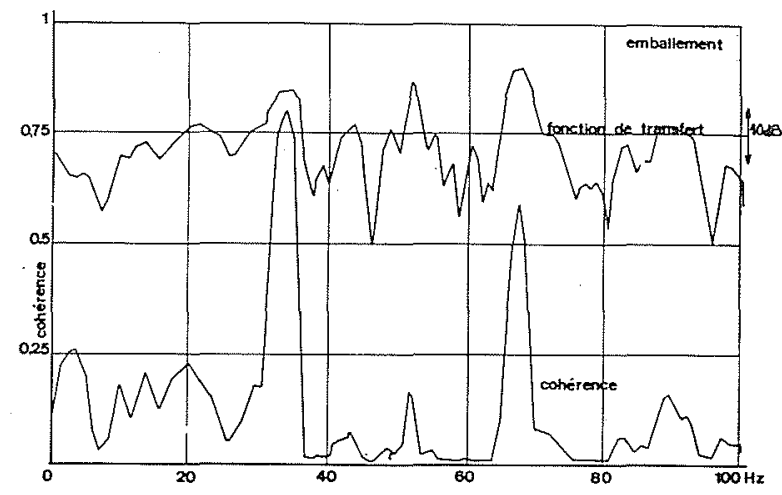

Figure 2 - Ste Croix. Fonction de transfert et de cohérence (Couple directrice/Pression entrefer).

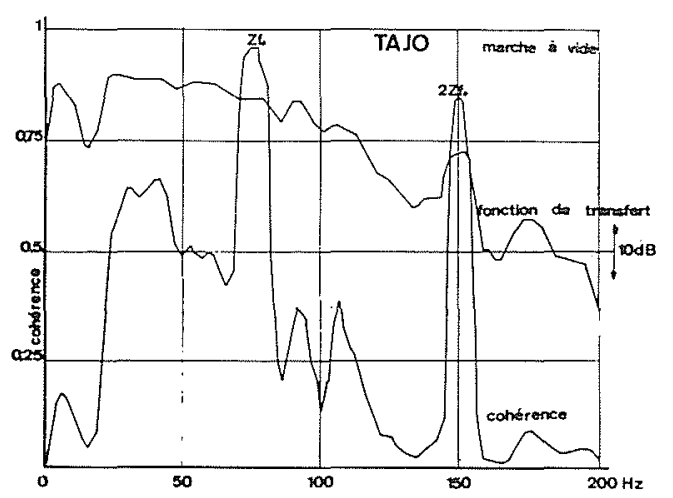

Figure 3 - Revin-Tajo : Fonction de transfert et de cohérence (Couple directrice/Pression entrefer). 
Nous avons porté sur la figure 4 avec:

$\Delta H:$ fluctuation de pression crête à crête ;

$n \quad$ : vitesse de rotation à l'emballement;

$D_{2}$ : diamètre sortie pompe de la roue.

la valeur des fluctuations de pressions réduites $\Delta H /\left(n D_{2}\right)^{2}$ en fonction de l'ouverture en turbine ramenée à l'ouverture optimale pour des machines de différentes vitesses spécifiques .

Nous observons que les courbes sont classées en première approximation, dans le même ordre que les vitesses spécifiques des machines, à savoir qu'à ouverture réduite constante, les fluctuations de pression dans l'entrefer augmentent quand la vitesse spécifique diminue.

Pour une turbine Francis de grande vitesse spécifique, les fluctuations sont beaucoup plus faibles.

La comparaison des résultats précédents est rendue difficile par suite du caractère très local de la mesure des pressions. Il paraît donc plus intéressant de s'attacher à l'analyse des couples qui intègre la totalité des pressions le long du profil.

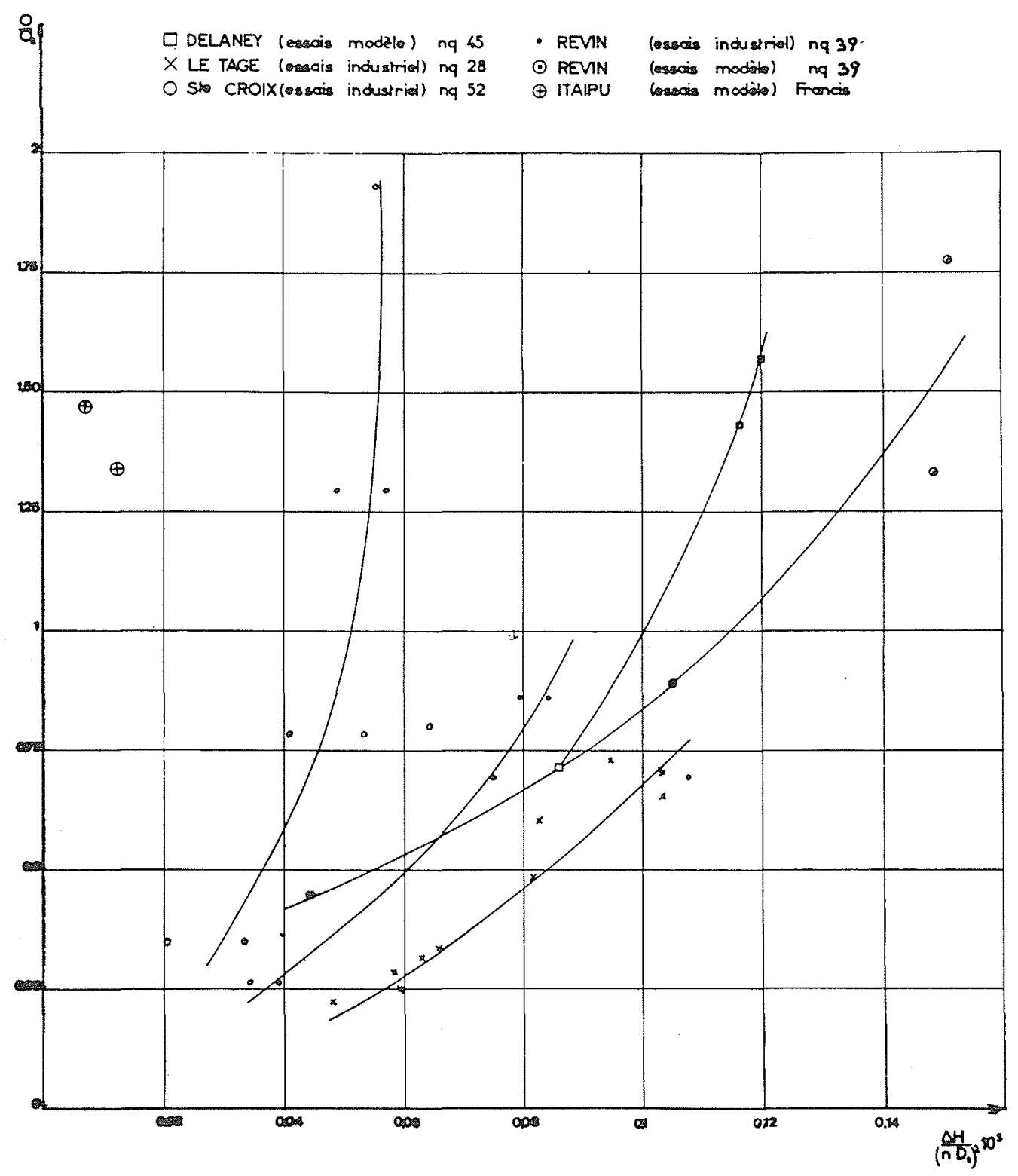

Figure 4 - Fluctuations réduites de pressions dans l'entrefer roue-distributeur. 


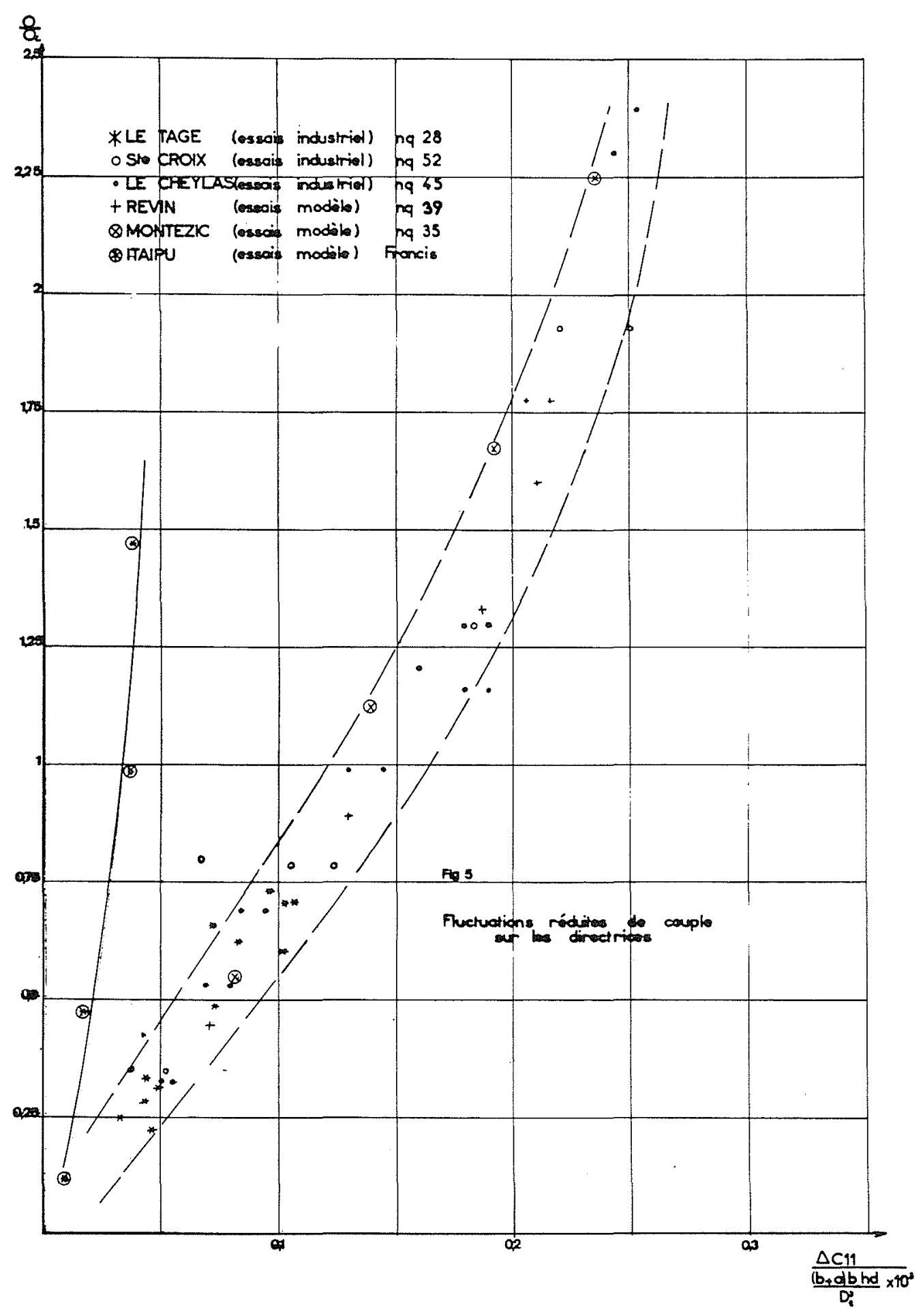

Figure 5 - Fluctuations réduites de couple sur les directrices. 


\section{Fluctuations de couple sur les directrices}

Les valeurs des fluctuations de couple sur les directrices dépendent essentiellement :

- de la forme du profil de la directrice,

- de la position du tourillon,

- de la hauteur du canal (liée à la vitesse spécifique)

- de l'entrefer roue-distributeur,

- du coefficient de cavitation

- et de divers paramètres liées à la géométrie de la roue.

Pour éliminer les facteurs liés à la position du tourillon et à la hauteur du canal, nous considérons le couple réduit ainsi défini

$$
K=\frac{\Delta C_{11}}{\frac{(b+a) b h d}{D_{2}^{3}}}
$$

avec

$$
\Delta C_{11}=\frac{\Delta C}{H D_{2}^{3}}
$$

et

$\Delta C:$ fluctuations de couple sur les directrices (crête à crête);

$H$ : chute nette;

$D_{2}$ diamètre sortie pompe de la roue ;

hd : hauteur des directrices;

$b$ : distance axe tourillon-sortie des directrices (sens turbine);

$a \quad$ : distance axe tourillon-entrée des directrices (sens turbine).

Nous avons porté sur la figure 5 les valeurs de ce couple réduit $K$ en fonction de l'ouverture 0 ramenée à l'ouverture au point optimal en turbine $\left(0_{0}\right)$ pour les différentes turbomachines.

Les résultats relatifs aux turbines-pompes se situent dans une bande étroite pour une plage relativement importante des vitesses spécifiques considérées

$$
\left(28<n_{q}<52\right)
$$

Il semblerait donc que les résultats obtenus soient peu affectés par la vitesse spécifique des machines.

Cependant le couple réduit prend en compte dans une certaine mesure la vitesse spécifique par l'intermédiaire de la hauteur hd du canal. Les valeurs relatives à la turbine Francis sont nettement décalées par rapport aux résultats des turbines-pompes.

$\mathrm{La}$ dispersion résiduelle des résultats devrait s'expliquer par les facteurs déjà cités :

- la forme des directrices;

- l'entrefer ;

- le coefficient de cavitation;

- la géométrie de la roue.

\section{Forme des directrices}

L'influence du profil ne semble pas primordiale puisque parmi les machines étudiées, se trouvent des directrices de forme très différente et qui se classent correctement dans le diagramme de la figure 5 .
Les deux profils extrêmes considérés sont représentés sur la figure 6.
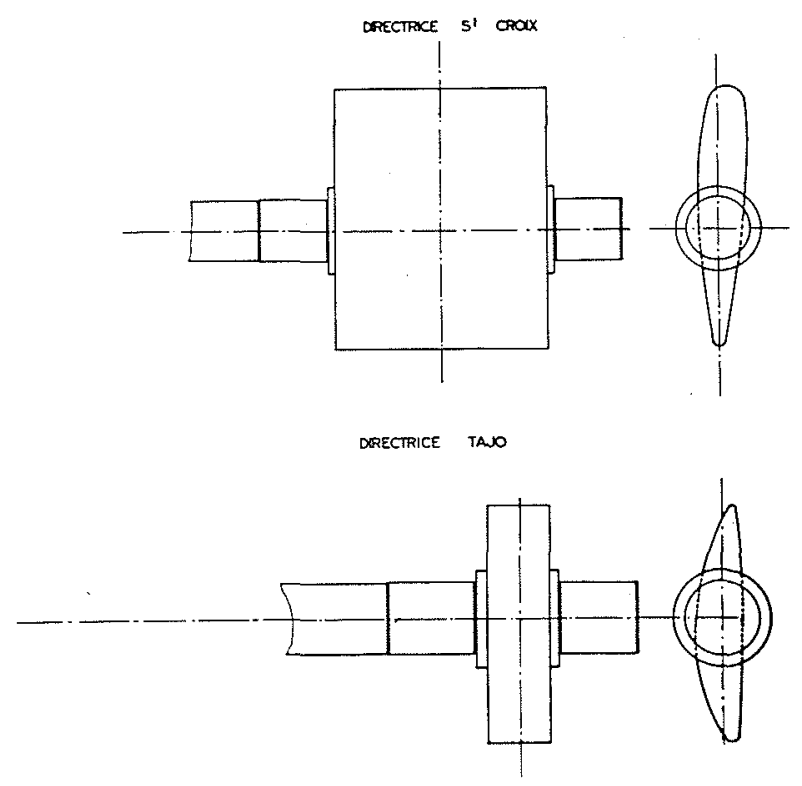

Figure 6 - Comparaison des profils des directrices de Ste Croix et du Tage.

\section{Entrefer}

Nous désignons par "entrefer" la distance (pour chaque ouverture du distributeur) entre la sortie des directrices et l'entrée de la roue (sens turbine) : $e=$ rayon sortie directrice - rayon entrée roue. Pour faciliter les comparaisons entre les différents essais, l'entrefer ainsi défini a été rapporté au rayon d'entrée roue (sens turbine).

Les variations peuvent être obtenues, soit par raccourcissement des directrices, soit par réduction du grand diamètre de la roue ("rognage"). Les résultats sur les fluctuations de couple sont alors différents.

Variation de l'entrefer par raccourcissement des directrices (Fig. 7)

Les fluctuations de couple ont été portées pour trois ouvertures du distributeur, avant et après raccourcissement des directrices.

L'influence de l'entrefer, importante à grande ouverture du distributeur, diminue pour une ouverture proche du point optimal en turbine et devient négligeable à faible ouverture.

Les résultats d'un calcul schématisant l'écoulement bidimensionnel, instationnaire et irrotationnel d'un fluide parfait incompressible dans une turbomachine sont portés sur cette même figure. Bien que les hypothèses soient relativement éloignées du cas qui nous intéresse, on peut remarquer que les valeurs obtenues donnent un ordre de grandeur des variations des fluctuations de couple en fonction de l'entrefer.

Variation de l'entrefer par "rognage de la roue" (Fig. 8)

L'influence du "rognage" de la roue n'est pas la même pour les trois ouvertures de distributeur consi- 
dérées. A grande ouverture, l'augmentation de l'entrefer se traduit par une diminution des fluctuations de couple, mais beaucoup plus faible que celle obtenue dans le cas précédent. Pour une ouverture proche de celle du point optimal, cette influence devient nulle alors que dans les cas précédent, elle restait encore sensible.

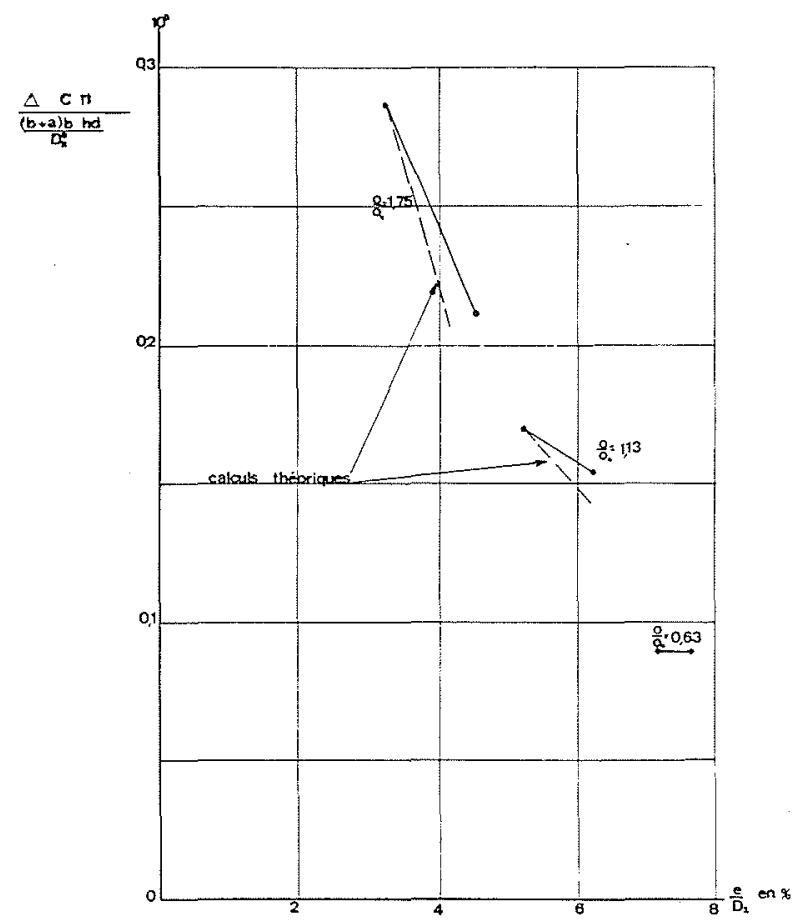

Figure 7 - Influence de l'entrefer relatif $e / D$ sur les pulsations de couple réduit. Variation d'entrefer obtenue par accourcissement du profil des directrices.

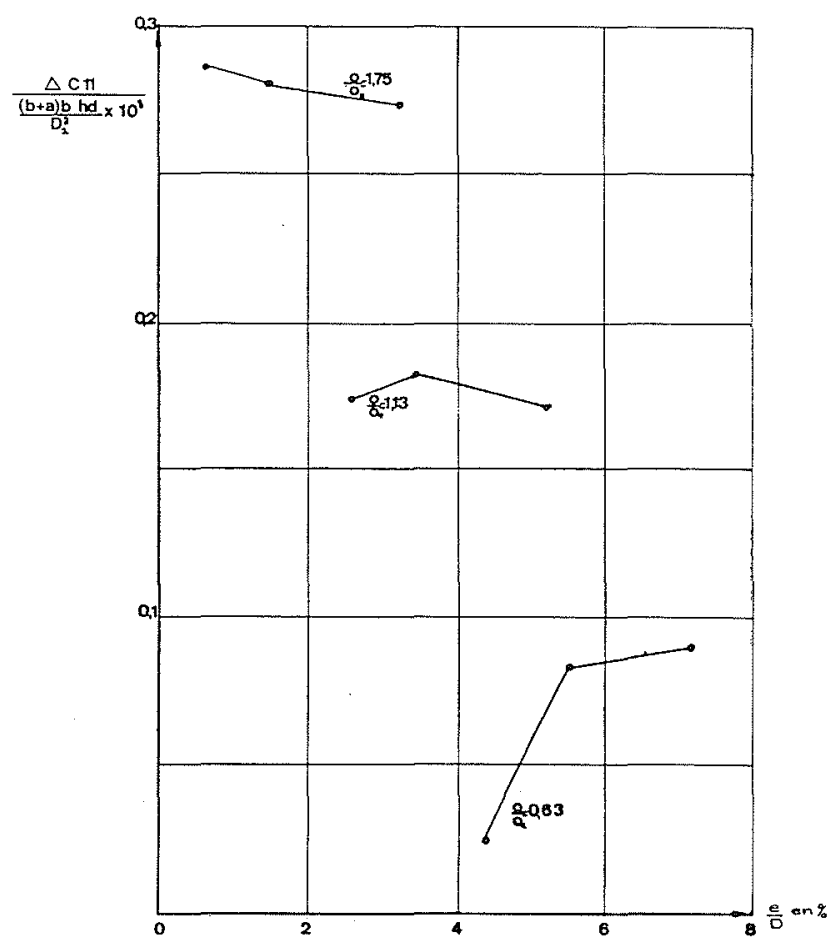

Figure 8 - Influence de l'entrefer relatif $e / D$ sur les pulsations de couple réduit. Variation d'entrefer obtenue par réduction du diamètre de roue $D_{2}$ (rognage).
Par contre à faible ouverture, elle s'inverse contrairement au cas précédent. Ainsi le rognage de la roue a moins d'influence sur les fluctuations de couple que le raccourcissement équivalent des directrices pour les grandes ouvertures de distributeur et il a un effet contraire pour les très faibles ouvertures.

Remarque : La part de l'influence de l'entrefer dans la dispersion des points de la figure 5 (variation du couple réduit $K$ en fonction de l'ouverture réduite) est très limitée, car il s'agit d'essais de machines industrielles ou de modèle en similitude pour lesquels l'entrefer a des valeurs réduites très proches.

\section{Coefficient de cavitation $\sigma$}

L'influence de $\sigma$ sur les fluctuations de couple a été étudiée en laboratoire. La figure 9 représente quelques résultats pour différentes ouvertures réduites du distributeur. On observe que les fluctuations de couple augmentent quand sigma diminue.

Cette augmentation est de l'ordre de $20 \%$ quand $\sigma$ est divisé par 2 .

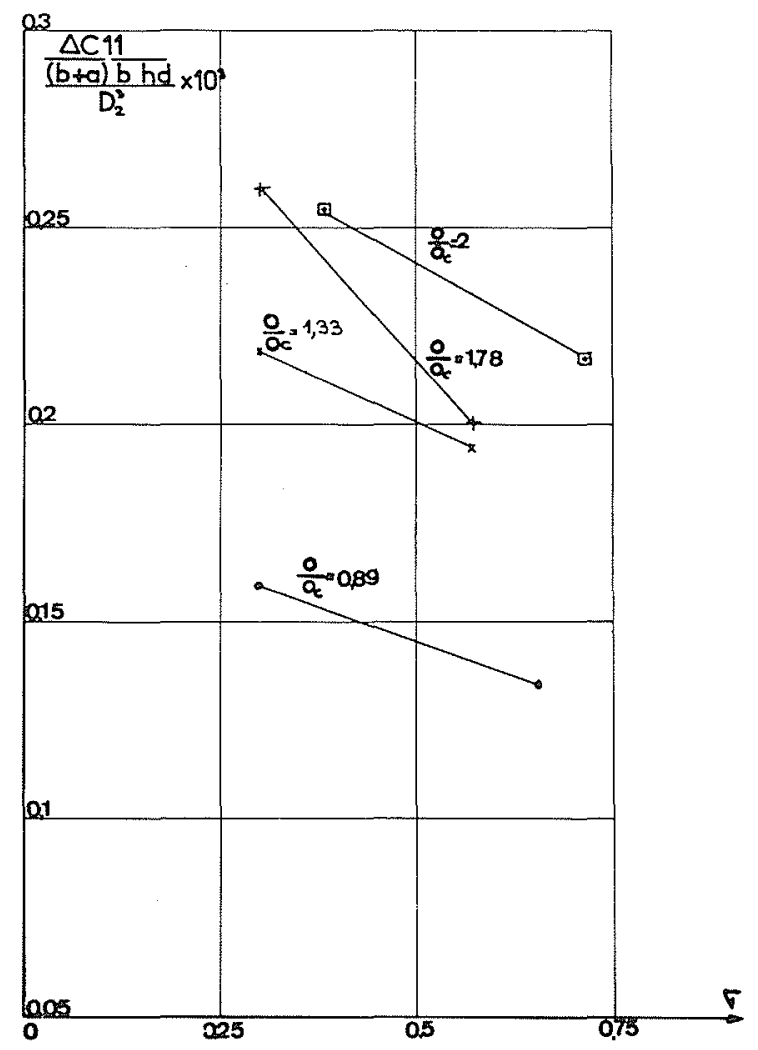

Figure 9 - Influence de $\sigma$ sur les fluctuations de couple sur les directrices.

\section{Géométrie de la roue}

Nous avons vu que l'accroissement de l'entrefer selon qu'il est réalisé, sur la partie fixe ou la partie mobile, a une influence différente sur le coefficient $K$ de fluctuation de couple.

Sur la partie fixe (distributeur) le phénomène est plus facilement analysable par le calcul. Par contre, l'effet du 
"rognage" de la roue suivant les ouvertures réduites doit modifier la structure interne de l'écoulement et il est alors difficile de schématiser le problème.

Il en résulte que l'analyse de l'influence des éléments constructifs d'une roue de turbine-pompe sur les fluctuations de couple à l'emballement est difficile à calculer.

Divers paramètres ont cependant été considérés :

- angle de sortie $\hat{\beta} 2$ des aubes,

- nombre $z$ d'aubes,

- angle de pendange de l'arête de sortie pompe,

- mais les résultats sont peu significatifs, en partie parce que l'amplitude des variations des paramètres envisagés restait faible dans nos expériences et ne permettait pas de dégager des lois suffisamment nettes.

\section{Conclusion}

Pour les machines considérées, il ressort que le paramètre le plus important qui intervienne dans les sollicitations du distributeur à l'emballement, soit l'angle d'injection de l'eau à l'entrée de la roue, c'est-à-dire l'ouverture du distributeur.

L'influence de l'entrefer et de la position du tourillon des directrices peut être estimée moyennant les hypothèses précédemment énoncées et les résultats obtenus sont conformes à l'expérience (à une réserve près, pour le "rognage" des roues).

En ce qui concerne les facteurs liés à la géométrie de la roue, leur influence n'a pas pu être décelée avec suffisamment de précision, leurs variations n'étaient pas assez significatives sur les machines étudiées.

\section{Discussion}

Président : S. CASACCI

M. le Président. - Avez-vous des observations sur cette communication?

M. DESMET. - Pour étudier l'influence de la pression dans l'entrefer sur le couple de la directrice, vous avez tracé la fonction de transfert couple directrice/pression entrefer, mais celle-ci présente une mauvaise cohérence sur la plupart des fréquences, sauf pour deux.

N'aurait-il pas été plus intéressant, de façon à mieux isoler les fréquences du spectre du couple sur les directrices, d'utiliser une fonction d'interspectre, pour mieux connaître la part de l'influence de la pression d'entrefer?

M. BOUSSUGES. - - La fonction de transfert figurant sur le graphique projeté n'a été donnée qu'à titre comparatif. Nous nous sommes surtout servi de la fonction cohérence $\gamma(f)$.

Nous aurions pu, comme vous le suggérez, utiliser l'interspectre $G x y(f)$. L'interspectre et la fonction de cohérence sont d'ailleurs liés par la relation

$$
\gamma^{2}(f)=\frac{|G x y(f)|^{2}}{G x x(f): G y y(f)}
$$

où $G x x(f)$ et $G y y(f)$ sont les fonctions d'autospectres respectivement des signaux $x$ et $y$ analysés.

Nous avons préféré utiliser la fonction de cohérence parce qu'elle présente l'avantage de caractériser directement la quantité d'énergie commune.

De plus, une cohérence faible peut provenir de signaux analysés eux-mêmes faibles donc plus ou moins noyés đans le bruit. Dans le cas actuel, nous pensons qu'il y a surtout le fait que la mesure de pression est ponctuelle alors que le couple sur les directrices intégrant les pressions autour du profil reste plus significatif.

C'est la raison pour laquelle nous nous sommes focalisés surtout sur les couples sollicitant les directrices.

M. LIESS. - Je voudrais poser une question :

Sur la première figure, vous avez montré une distribution calculée des vitesses à l'emballement. Avez-vous tenu compte des parties avec cavitation?
M. BOUSSUGES. - C'est un calcul en fluide parfait. Il s'agit d'un schéma très général, dans le but d'appréhender l'importance du gradient de pression du côté de la face " $a$ " de l'aube. Ce diagramme n'a pas d'autre signification que de faire prendre conscience des variations rapides des pressions de l'écoulement à l'entrée de la roue qui provoquent le décollement đ'entrée.

$M$. le Président. - Y a-t-il d'autres questions ?

M. GUITON. - La même chose avait été demandée en marche à vide; je ne sais pas si cela a été fait.

M. BORCIANI. - Sur le diagramme, figure 10 , j'ai reporté les résultats de mesures à l'emballement, effectués sur une turbine-pompe de $n_{q}=42$ (centrale de Chaplina en Yougoslavie). Les valeurs réduites des fluctuations de pression ont une bonne cohérence avec celles mesurées par les auteurs.

En ce qui concerne les turbines Francis, nous n'avons pas jusqu'à maintenant effectué de mesure des fluctuations de pression dans l'entrefer pour les machines industrielles.

M. BOUSSUGES. - La turbine Francis est en quelque sorte un témoin de l'écart qu'il y a entre turbines-pompes et turbines. Les résultats portés sur le diagramme correspondent à des essais sur modèle.

M. le Président. - La turbine Francis est une référence. Elle est de $n_{s}=240$ environ, c'est-à-dire qu'elle ne coupe pas son débit ; il n'y a pas de variations de débit en fonction de la vitesse. Donc, elle n'a rien à voir aver une turbine-pompe.

M. BOUSSUGES. - Il y a quand même une certaine continuité.

M. le Président. - Sur une turbine de faible vitesse spécifique, nous avons mesuré des variations de couple à l'emballement importantes, à cause du diamètre et à cause du fait que la turbine coupe son débit à l'emballement.

En somme, les fluctuations de couple et de pression sont dues au fait que la turbine constitue un vannage - un mauvais vannage - qui empêche l'eau de traverser la roue et que, par conséquent, il $\mathrm{y}$ a toute une série de recirculations entre les aubes et les directrices. 
M. HENRY. - Je voudrais ajouter un bref commentaire à ce que vous venez de dire. On a essayé des machines toutes proches qui coupaient considérablement le débit à l'emballement. M. $B O R C I A N I$ a projeté ce matin une Francis de $n_{q}=65$ qui a son débit complètement coupé globalement.

M. le Président. - Je sais qu'une machine de grande vitesse spécifique ne coupe pas son débit à l'emballement; le débit reste à peu près constant et n'est pas influencé par la vitesse. I est un peu étonnant qu'une Francis de ce " $n_{s}$ " - là coupe son débit à l'emballement, mais ce n'est peut être pas impossible.

M. BOUSSUGES. - C'est probablement que le tracé est d'une coupe méridienne particulière.

M. HENRY. - Non, cela dépend du $\sigma$.
M. ROTH. - Vous avez parlé des aubes anormalement sollicitées. En avez-vous tenu compte pour l'emplacement de l'aube directrice par rapport à la bâche diffuseur, par rapport au bec de bâche ou loin du bec de bâche?

M. BOUSSUGES. - Ces résultats sont des résultats moyens sur un certain nombre d'essais localisés.

M. ROTH. - Vous avez parlé de sollicitations plus fortes à l'emballement en position désynchronisée d'une directrice par rapport aux directrices voisines. Avez-vous fait des essais à ce sujet?

$M$. BOUSSUGES. - Oui, mais on n'a pas pu en tenir compte sur les diagrammes parce que cela aurait nettement compliqué l'exposé.

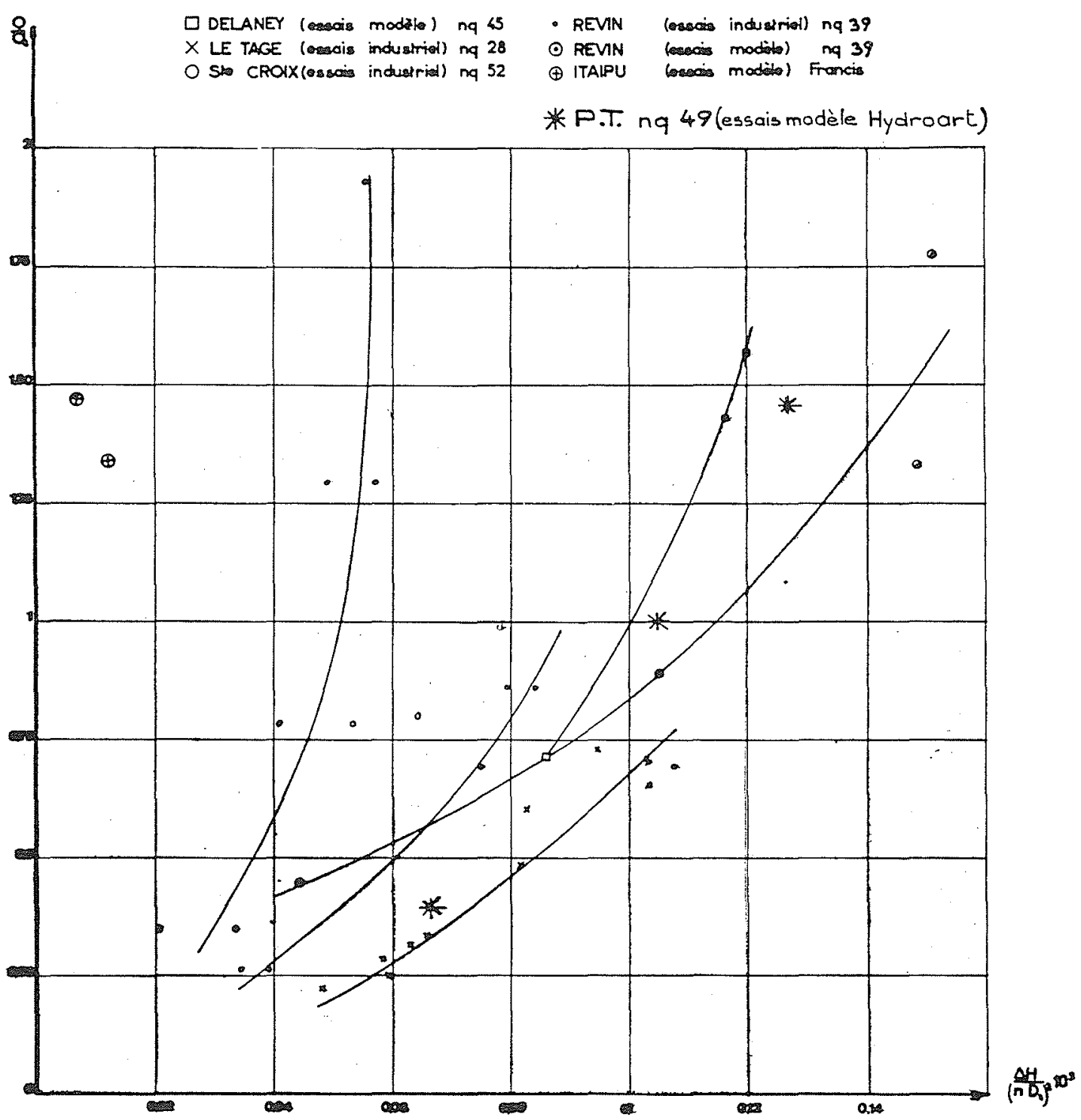

Figure 10 - Fluctuations réduites de pression dans l'entrefer roue-distributeur - Résultats des mesures à l'emballement sur une turbinepompe $n_{q}=42$ de la centrale de Chaplina en Yougoslavie. 
M. ROTH. - Les efforts sont peut être plus grands?

M. BOUSSUGES. - Il sont en effet plus grands en désynchronisation. Je m'excuse de ne pas l'avoir précisé dans l'exposé, il s'agit de toutes les directrices synchronisées.

M. le Président. - Elles ont toutes le mème angle.

M. BOUSSUGES, - Le système est axi-symétrique.

M. le Président. - De toutes façons, on ne peut admettre pratiquement qu'une désynchronisation limitée, parce que, s'il y a une désynchronisation assez forte, on est tenu de fermer le distributeur le plus rapidement possible pour éviter que la machine soit trop sollicitée. Cette désynchronisation limitée ne doit pas avoir beaucoup d'influence sur les pulsations.

Je vous fais remarquer que, pour les mesures de pulsation de couple sur les directrices, il faut, bien entendu, que, sur modèle, la fréquence propre de torsion des directrices soit très loin des efforts qu'on veut mesurer; sinon, on va mesurer une amplification due au modèle lui-même. Par conséquent, il faut que la fréquence propre de torsion de la directrice soit très élevée par rapport aux fréquences des pulsations de couple que l'on mesure.

M. GUITON. - Il y a quelques années, on avait vu un film présenté par ESCHER WYSS sur l'écoulement dans le distri- buteur à différents régimes. 11 m'avait semblé voir - mais c'était très rapide - un décollement tournant dans la distributeur à certains régimes anormaux. Avez-vous constaté de telles choses au voisinage de l'emballement ou de la marche à vide ?

M. JARRIAND. - Le décollement tournant, on a cru l'avoir saisi dans une installation industrielle au Tajo, mais on ne l'a jamais saisi en modèle réduit.

M. le Président. - On ne l'a jamais visualisé sur modèle. Les Japonais ont publié des visualisations sur turbine-pompe et il semble qu'il y a un décollement tournant. Mais nous ne l'avons jamais visualisé sur modèle, en ce qui nous concerne.

S'il n'y a plus de questions, il me reste à clore la session.

Je vous demande d'excuser M. le Président du Comité Technique de la Société Hydrotechnique de France, M. CAZENAVE, qui est retenu par la réunion du Bureau Technique de la Société.

Je tiens à remercier tous les participants à cette session de la S.H.F., et je voudrais faire une mention particulière pour tous nos collègues étrangers qui ont fait l'effort d'une part de se déplacer, d'autre part d'accomplir un travail considérable, d'avoir apporté une contribution véritablement importante et, pour eux dont la langue maternelle n'est pas le français, d'avoir bien voulu s'exprimer dans cette langue. 